\title{
CDISC SDTM Device-In-Use Response Terminology
}

National Cancer Institute

\section{Source}

National Cancer Institute. CDISC SDTM Device-In-Use Response Terminology. NCI Thesaurus. Code C129938.

Terminology associated with the device-in-use response codelist of the Clinical Data Interchange Standards Consortium (CDISC) Study Data Tabulation Model (SDT M). 